\title{
$\mathrm{RuO}_{2}$ Monolayer: A Promising Bi-Functional Catalytic Material for
}

\section{Non-Aqueous Lithium-Oxygen Batteries}

\section{Supporting Information}

\author{
Le Shi, Ao Xu, Tianshou Zhao*
}

Department of Mechanical and Aerospace Engineering The Hong Kong University of Science and Technology

Clear Water Bay, Kowloon, Hong Kong, China

Table. S1 Optimized lattice parameters of $\mathrm{Li}_{2} \mathrm{O}_{2}, \mathrm{RuO}_{2}$ monolayer and rutile $\mathrm{RuO}_{2}$

\begin{tabular}{cccc}
\hline & $\mathrm{a}(\AA)$ & $\mathrm{b}(\AA)$ & $\mathrm{c}(\AA)$ \\
\hline $\mathrm{Li}_{2} \mathrm{O}_{2}\left(P 6_{3} / m m c\right)$ & 3.14 & 3.14 & 7.65 \\
\hline $\mathrm{RuO}_{2} \mathrm{monolayer}(R \overline{3} m)$ & 2.93 & 2.93 & -- \\
\hline Rutile $\mathrm{RuO}_{2}\left(P 4_{2} / m n m\right)$ & 4.52 & 4.52 & 3.11 \\
\hline
\end{tabular}

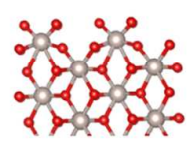

O-rich

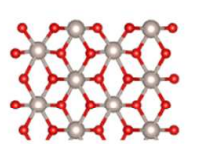

stoichiometric

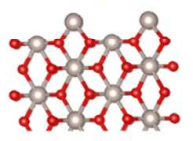

Ru-rich

$\{001\}$

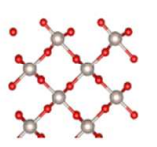

O-rich

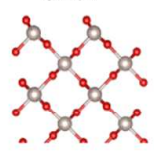

stoichiometric
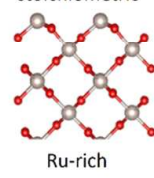

$\{100\}$

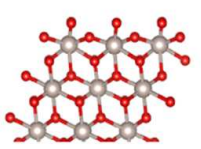

O-rich

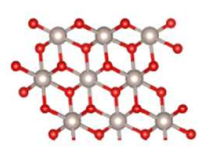

stoichiometric
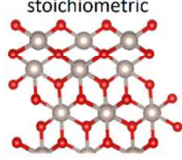

Ru-rich

$\{101\}$

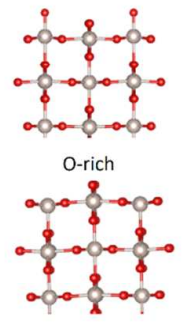

stoichiometric

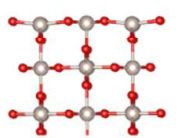

Ru-rich

\{110\}

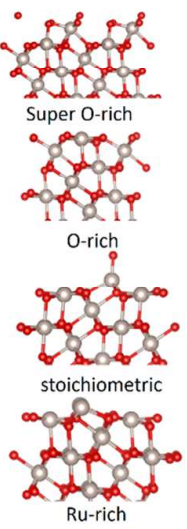

\{111\}

Ruthenium atom

Oxygen atom

Fig. S1 The orientations and terminations of rutile $\mathrm{RuO}_{2}$ considered for the Wulff construction. 


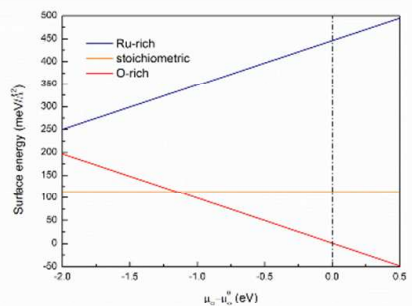

$\{001\}$

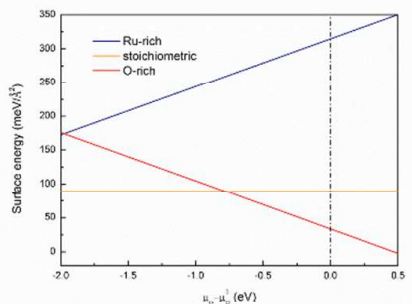

$\{100\}$

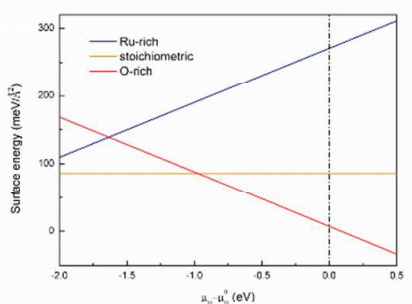

$\{101\}$
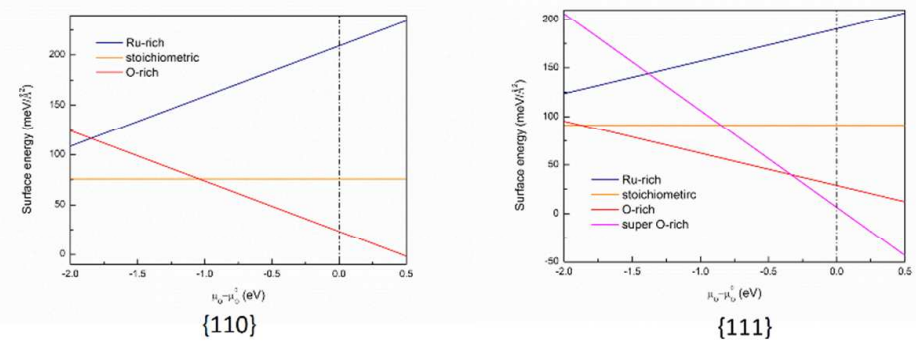

Fig. S2 Surface energies of different orientations and terminations of rutile $\mathrm{RuO}_{2}$ under different oxygen chemical potentials

(a)

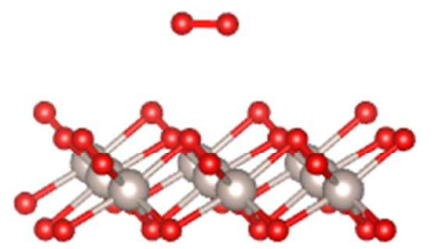

Ruthcnium atom (b)
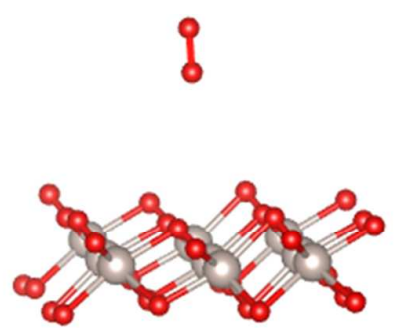

- Oxygen atom

Fig. S3 Optimized geometry for oxygen molecular adsorbed onto the surface of $\mathrm{RuO}_{2}$ monolayer. Two initial geometries were considered ( $a$ and $b$ ). The oxygen-oxygen bond lengths in both cases are $1.22 \AA$ (The bond length for free oxygen molecular is $1.21 \AA)$. 
(a)

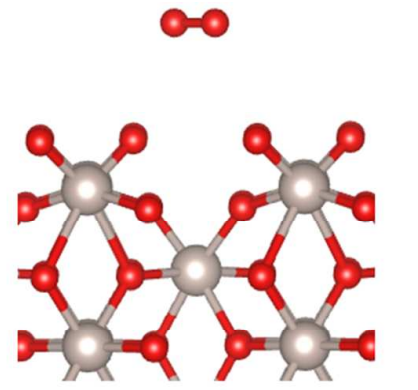

Q Ruthenium atom (b)

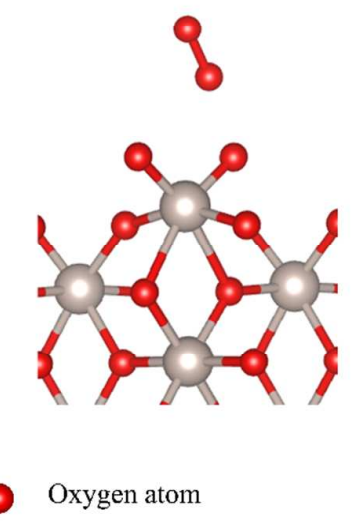

Fig. S4 Optimized geometry for oxygen molecular adsorbed onto rutile $\mathrm{RuO}_{2}\{001\}$ surface. Two initial geometries were considered ( $a$ and $b$ ). The oxygen-oxygen bond lengths for both cases are $1.21 \AA$ (The bond length for free oxygen molecular is 1.21 $\AA$ ).

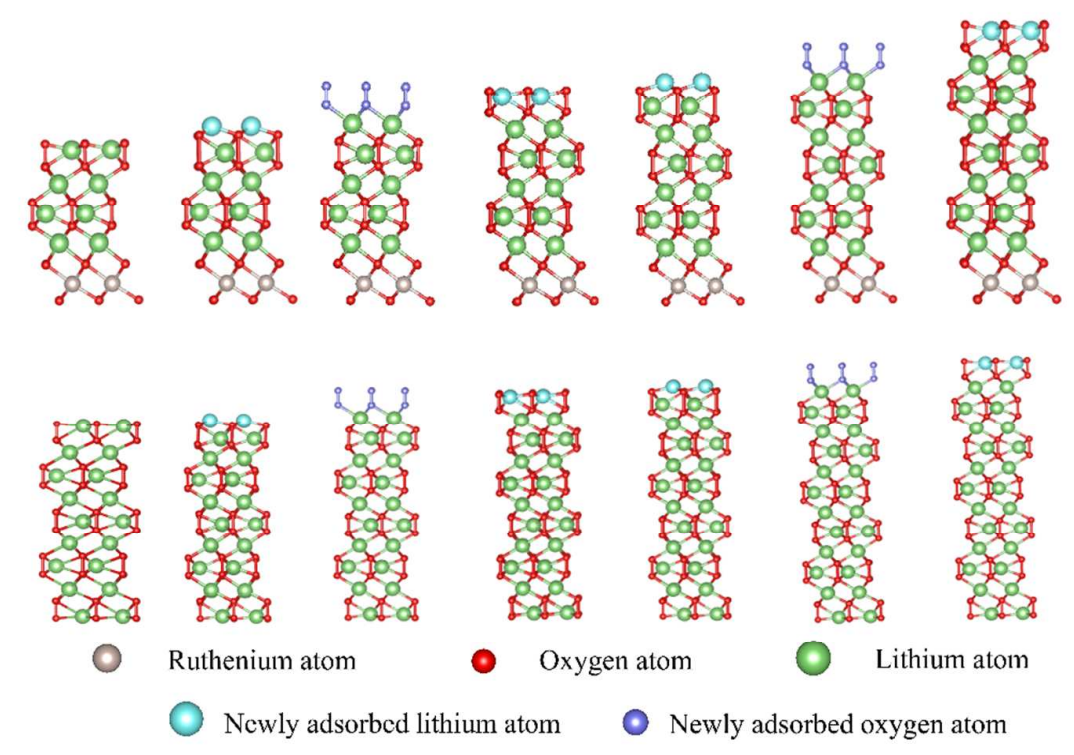

Fig. S5 Optimized geometry of discharge process happened on $\mathrm{Li}_{2} \mathrm{O}_{2}\{0001\}$ surface with and without $\mathrm{RuO}_{2}$ monolayer. 


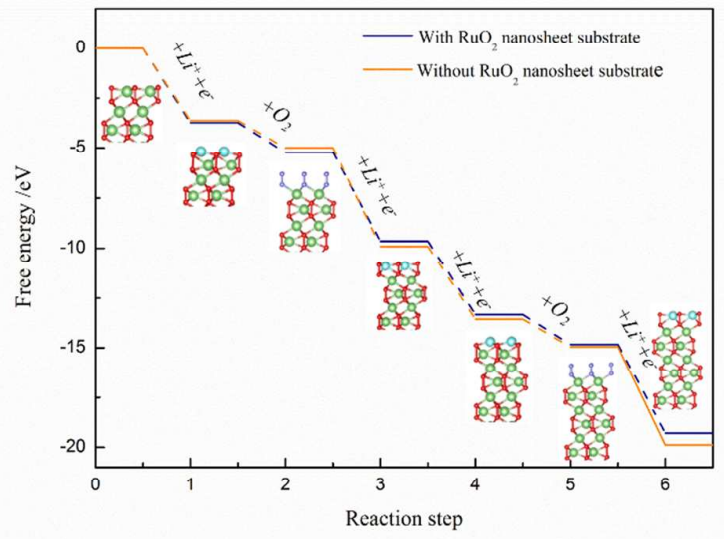

Ruthenium atom

- Oxygen atom

Lithium atom

Newly adsorbed lithium atom

- Newly adsorbed oxygen atom

Fig. S6 Energy profiles for the discharge process happened on the $\mathrm{Li}_{2} \mathrm{O}_{2}\{0001\}$ surface with and without $\mathrm{RuO}_{2}$ monolayer

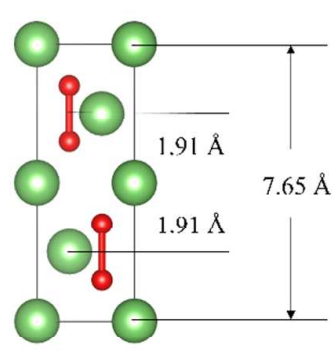

Ruthenium atom

- Oxygen atom

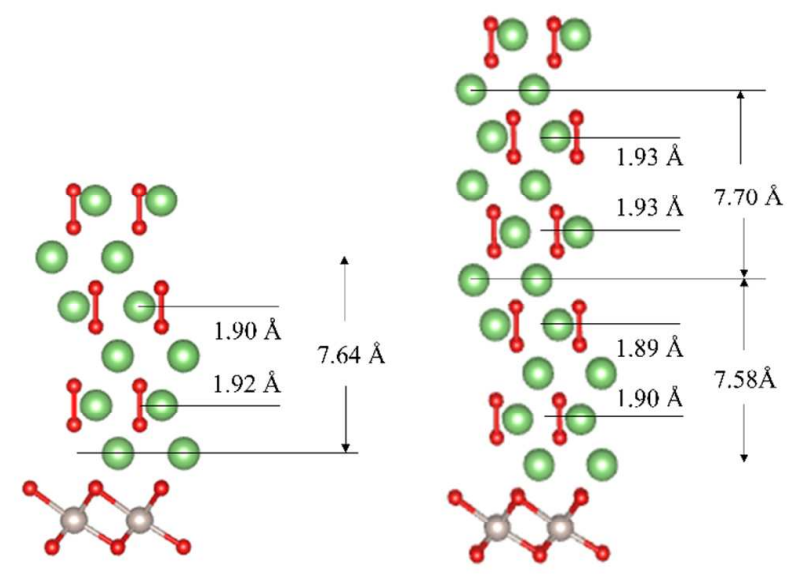

Lithium atom

Fig. S7 Optimized lattice parameters of $\mathrm{Li}_{2} \mathrm{O}_{2}$ before and after adsorption onto $\mathrm{RuO}_{2}$ monolayer. 


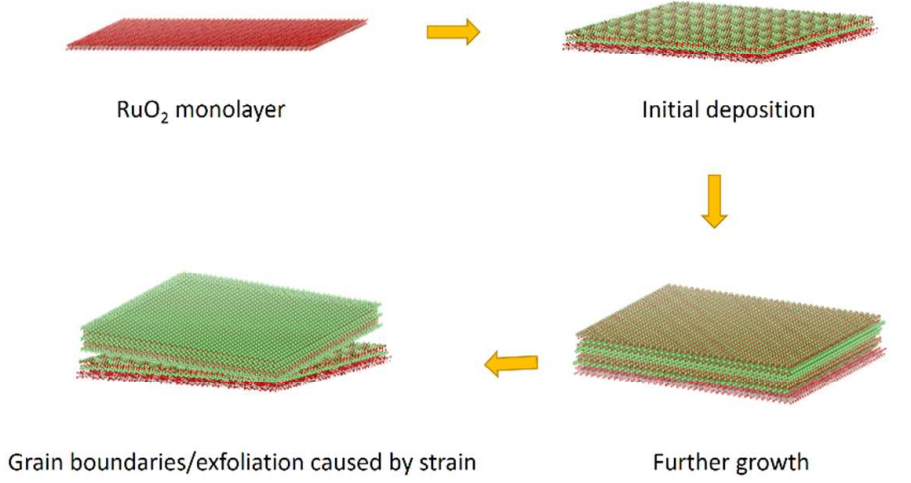

Fig. S8 Illustration for proposed formation mechanism for the assembled thin disc morphology of $\mathrm{Li}_{2} \mathrm{O}_{2}$

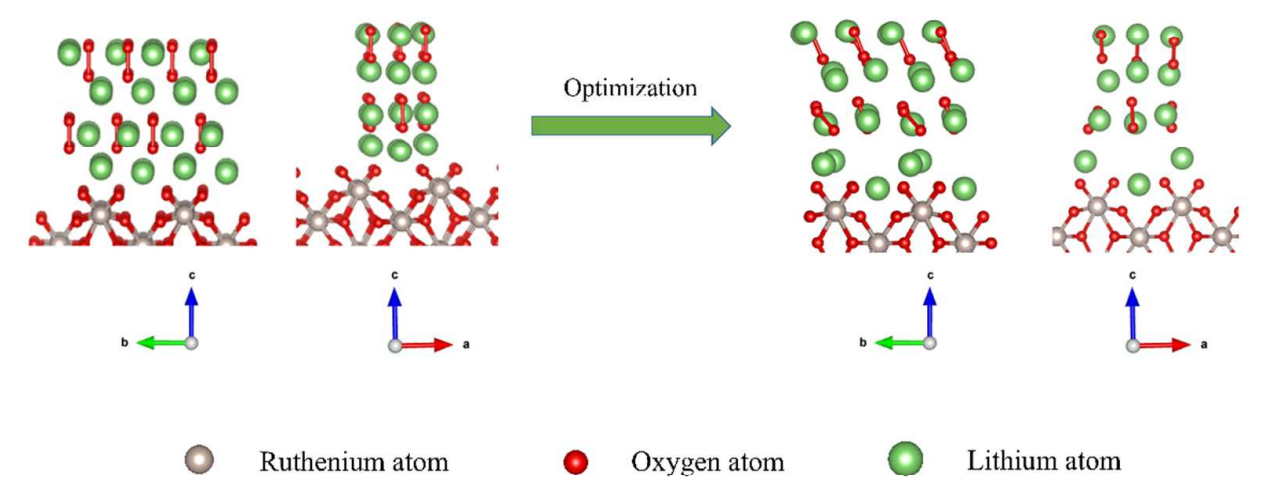

Fig. S9 The geometries of the interfacial model of rutile $\mathrm{RuO}_{2}\{001\}$ surface and $\mathrm{Li}_{2} \mathrm{O}_{2}\{0001\}$ surface before and after optimization. The white, red and green balls correspond to ruthenium, oxygen and lithium atoms respectively. 